Acta Crystallographica Section D

Biological Crystallography

ISSN 0907-4449

\section{Comparing crystallographic and solution structures of nitrogenase complexes}

\section{J. Günter Grossmann, ${ }^{a}$ S. Samar Hasnain, ${ }^{\mathrm{a}}{ }^{*}$ Faridoon $\mathrm{K}$. Yousafzai, ${ }^{\text {b }}$ Barry E. Smith, Robert R. Eady, ${ }^{b}$ Hermann Schindelin, ${ }^{\mathrm{C}}+$ Caroline Kisker, ${ }^{\mathrm{C}} \neq$ James B. Howard, ${ }^{d}$ Hirotsugu Tsuruta, ${ }^{e}$ Jurgen Muller ${ }^{\mathrm{f}}$ and Douglas C. Rees ${ }^{\mathrm{c}, \mathrm{g}}$}

${ }^{a}$ CLRC Daresbury Laboratory, Synchrotron Radiation Department, Warrington, Cheshire WA4 4AD, England, bitrogen Fixation Laboratory, John Innes Centre, Colney, Norwich NR4 7UH, England, 'Division of Chemistry and Chemical Engineering, California Institute of Technology, Pasadena, CA 91125, USA dDepartment of Biochemistry, University of Minnesota, Minneapolis, Minnesota 55455, USA, ${ }^{\mathbf{e}}$ Stanford Synchrotron Radiation Laboratory, Stanford, CA 94305, USA, ' Max Delbrück Center for Molecular Medicine, D-13092 Berlin, Germany, and ${ }^{\mathbf{g}}$ Howard Hughes Medical Institute, California Institute of Technology, Pasadena, CA 91125, USA

+ Present address: Department of Biochemistry and Cell Biology, SUNY Stony Brook, NY 11794-5215, USA.

‡ Present address: Department of Pharmacological Sciences, SUNY Stony Brook, NY 11794-8651, USA.

Correspondence e-mail: s.hasnain@dl.ac.uk

Received 19 January 1999

Accepted 8 March 1999
Nitrogenase, the enzyme responsible for biological nitrogen fixation, catalyses the ATPdependent reduction of dinitrogen to ammonia (Howard \& Rees, 1994; Burgess \& Lowe, 1996). The X-ray crystal structures of the homodimeric $\mathrm{Fe}$ protein (Georgiadis et al., 1992) and the $\alpha_{2} \beta_{2}$ tetrameric MoFe protein (Kim \& Rees, 1992) have been determined. The $\mathrm{Fe}$ protein contains a single [4Fe-4S] cluster located at the dimer interface, while the $\mathrm{MoFe}$ protein contains two copies each of two different redox centres: the $\mathrm{P}$ clusters and FeMo cofactor. The $\mathrm{P}$ clusters are located at the interface of the $\alpha \beta$ subunits and the FeMo cofactor centres are buried in the $\alpha$ subunits some $20 \AA$ from the $\mathrm{P}$ clusters. It has been proposed that ATP hydrolysis by the $\mathrm{Fe}$ protein in the electron-transfer complex results in a conformational change, which allows electron transfer from the $[4 \mathrm{Fe}-4 \mathrm{~S}]$ centre of the Fe protein to the FeMo cofactor by way of the $\mathrm{P}$ cluster of the MoFe protein (Howard \& Rees, 1994).

The formation of a transient electrontransfer complex between the MoFe protein (Kp1 or Av1, where Kp refers to Klebsiella pneumoniae and Av refers to Azotobacter vinelandii) and the $\mathrm{Fe}$ protein ( $\mathrm{Kp} 2$ or $\mathrm{Av} 2)$ is an essential feature of the mechanism of nitrogenase. The two-component protein complex of nitrogenase is stabilized by aluminium tetrafluoride $\left(\mathrm{AlF}_{4}\right)$ in combination with ADP (Renner \& Howard, 1996; Duyvis et al., 1996), which traps an inactive transition-state complex. A low- resolution $(\sim 15 \AA)$ structure of $\mathrm{Kp} 1 \cdot\left(\mathrm{ADP} \cdot \mathrm{AlF}_{4}^{-} \cdot \mathrm{Kp} 2\right)_{2}$ was determined (Grossmann et al., 1997) using solution X-ray scattering data and was compared with the docking model (Kim \& Rees, 1992) based on the crystallographic structures of the $\mathrm{Av}$ component proteins. It was clear from this comparison that the Fe protein Kp2 undergoes a substantial conformational rearrangement in the complex, with $\mathrm{Kp} 1$ remaining essentially unaltered. Since then, the crystal structure of Av1.(ADP. $\left.\mathrm{AlF}_{4}^{-} \cdot \mathrm{Av} 2\right)_{2}$ has been determined at $3 \AA$ resolution (Schindelin et al., 1997), establishing that the docking site for the Fe protein is on the surface of the MoFe protein near the $\mathrm{P}$ cluster and detailing the conformational changes for the Fe protein in the complex. On average, a $4.1 \AA$ r.m.s. deviation in $\mathrm{C}^{\alpha}$ positions occurs between the free and complexed forms of Av2, as a result of an $\sim 13^{\circ}$ rigid-body rotation of each monomer toward the $\mathrm{Fe}$ protein dimer interface and localized structural changes in each subunit.

X-ray scattering data sets have been collected at the Daresbury SRS from further purified $\mathrm{Kp} 1 \cdot\left(\mathrm{ADP} \cdot \mathrm{AlF}_{4}^{-} \cdot \mathrm{Kp} 2\right)_{2}$ and at the Stanford Synchrotron Radiation Laboratory (SSRL) from Av1.(ADP.AlF $\left.{ }_{4}^{-} \cdot \mathrm{Av} 2\right)_{2}$ since the publication of the original low-resolution structure of this complex (Grossmann et al., 1997). The results given in Grossmann et al. (1997) refer to measurements where a small contribution ( $\sim 3-5 \%$ ) of free (unbound) $\mathrm{Kp} 2$ was still present in the solution. Since then, the purification of the transition-state complex has been improved and a cleaner complex sample was prepared, which is the subject of this report. This accounts for the slight differences (Figs. $2 a$ and $2 b$ ) compared with the molecular envelope presented initially. Although the scattering data were independently measured for two different complexes with different experimental set-ups, the scattering curves for the two complexes agree very closely, indi-

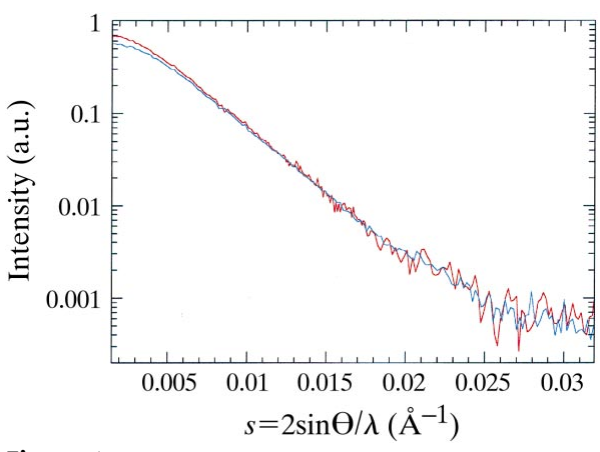

Figure 1

Comparison of solution X-ray scattering profiles of the ADP.AlF-stabilized nitrogenase complexes from $A$. vinelandii (blue) and $K$. pneumoniae (red). The scattering data for $\mathrm{Kp} 1 \cdot\left(\mathrm{ADP} \cdot \mathrm{AlF}_{4}^{-} \cdot \mathrm{Kp} 2\right)_{2}$ were collected at the SRS at Daresbury on sample concentrations of $1-5 \mathrm{mg} \mathrm{ml}^{-1}$ (data of $2 \mathrm{mg} \mathrm{ml}^{-1}$ shown), while the data for Av1.(ADP.AlF $4 \cdot A v 2)_{2}$ complex were collected at the Stanford Synchrotron Radiation Laboratory on sample concentrations of $2-15 \mathrm{mg} \mathrm{ml}^{-1}$ $\left(6 \mathrm{mg} \mathrm{ml}^{-1}\right.$ shown, which exhibits better statistics in the outermost scattering region). Concentration-dependent measurements are necessary owing to interference effects in the very low angle region for this complex (as seen here). Consequently, the final scattering profile is obtained by an extrapolation to zero concentration. The quality of agreement for the two scattering curves measured at different experimental facilities is remarkable; the slight deviation at the very low scattering angle primarily arises from slight aggregation effects owing to the higher concentrations of the Av complex. 


\section{scientific comments}
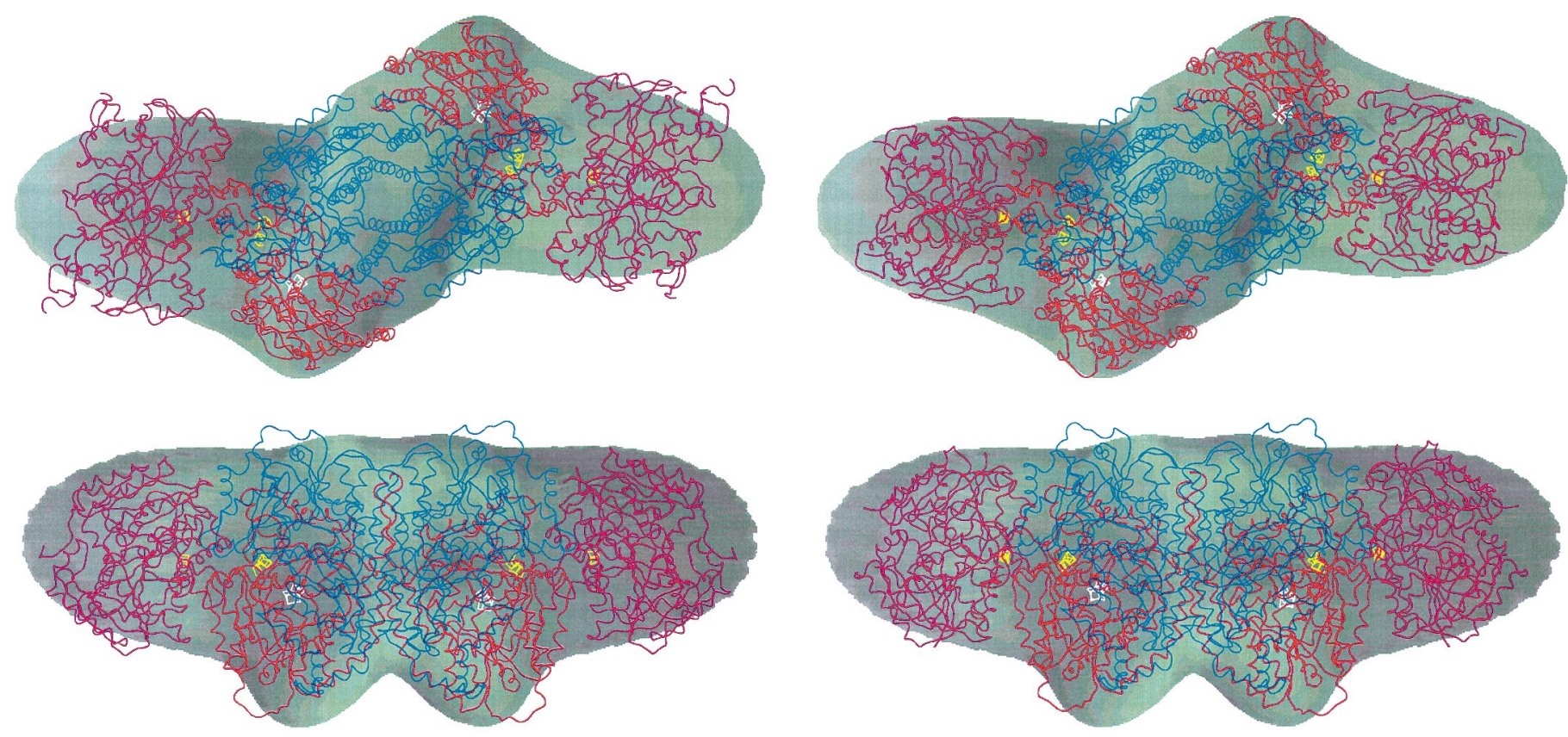

(a)

(b)

Figure 2

Molecular shape of the Kp transition-state complex (shaded in green) for the multipole order $L=6$ with superimposed ribbons of $(a)$ the docking model based on crystal structues of individual Av1 and Av2 proteins and $(b)$ the crystal structure of the Av1·(ADP·AlF-- $\left.\cdot \mathrm{Av}_{2}\right)_{2}$ complex. In each case two views are shown: one looking down the twofold symmetry axis and the other rotated by $90^{\circ}$ around the horizontal axis. Significant changes in the Fe protein (magenta ribbon) are clearly evident from the lowresolution molecular shape. For the sake of clarity the locations of FeS clusters (yellow) and MoFe centres (white) are highlighted.

cating that they possess similar solution structures. From the new scattering data for the Kp1·(ADP·AlF - - Kp2) 2 complex (Fig. 1), a low-resolution molecular structure has been obtained, which is shown superimposed on the docking model (Fig. $2 a$ ) and crystal structure (Fig. 2b) of the Av1.(ADP.AlF $\left.{ }_{4}^{-} \cdot \mathrm{Av} 2\right)_{2}$ complex. Procedures concerning the shape calculations from solution-scattering profiles are described elsewhere in more detail (Hao et al., 1999; Grossmann \& Hasnain, 1997; Grossmann et al., 1997; Svergun et al., 1995, 1997). R factors between the observed and calculated scattering data of 0.284 and 0.080 were obtained using the atomic coordinates of the rigid-body docked computer model and the crystal structure of the Av complex, respectively. This reduction in $R$ factor is consistent with similar $\mathrm{Fe}$ protein conformational changes taking place in the complexes for both species, in agreement with observations that the Av2-Kp1 and Kp2-Av1 heterocomplexes are fully active (Emerich et al., 1981).
The agreement between scattering curves of the complex from two different species strongly suggests that their respective transition-state complexes are very similar, and that the good fit between the crystallographic model and the model deduced from scattering data alone (albeit at low resolution) suggests the complex is the same in both.

The work was supported by the EPSRC/ BBSRC Biomolecular Science grant GR/J54512 and by a grant from the NIH. The small-angle scattering facility at SSRL is supported by DOE and NIH. We are also grateful for the provision of facilities at CCLRC Daresbury Laboratory and to Dr Loretta Murphy for her help and interest. We acknowledge helpful comments from the referees.

\section{References}

Burgess, B. K. \& Lowe, D. J. (1996). Chem. Rev. 96, 2983-3011.
Duyvis, M. G., Wassink, H. \& Haaker, H. (1996). FEBS Lett. 380, 233-236.

Emerich, D. W., Hageman, R. V. \& Burris, R. H. (1981). Adv. Enzymol. 52, 1-22.

Georgiadis, M. M., Komiya, H., Chakrabarti, P., Woo, D., Kornuc, J. J. \& Rees, D. C. (1992). Science, 257, 1653-1659.

Grossmann, J. G. \& Hasnain, S. S. (1997). J. Appl. Cryst. 30, 770-775.

Grossmann, J. G., Hasnain, S. S., Yousafzai, F. K. Smith, B. E. \& Eady, R. R. (1997). J. Mol. Biol. 266, 642-648.

Hao, Q., Dodd, F. E., Grossmann, J. G. \& Hasnain, S. S. (1999). Acta Cryst. D55, $243-$ 246.

Howard, J. B. \& Rees, D. C. (1994). Annu. Rev. Biochem. 63, 235-264.

Kim, J. \& Rees, D. C. (1992). Nature (London), 360, 553-560.

Renner, K. A. \& Howard, J. B. (1996). Biochemistry, 35, 5353-5358.

Schindelin, H., Kisker, C., Schlessman, J. L., Howard, J. B. \& Rees, D. C. (1997). Nature (London), 387, 370-376.

Svergun, D. I., Barberato, C. \& Koch, M. H. J. (1995). J. Appl. Cryst. 28, 768-773.

Svergun, D. I., Volkov, V. V., Kozin, M. B., Stuhrmann, H. B., Barberato, C. \& Koch, M. H. J. (1997). J. Appl. Cryst. 30, 798802. 